\title{
Dynamical ages of the young local associations with Gaia (Corrigendum)
}

\author{
N. Miret-Roig ${ }^{1,2}$, T. Antoja ${ }^{1}$, M. Romero-Gómez ${ }^{1}$, and F. Figueras ${ }^{1}$ \\ ${ }^{1}$ Institut de Ciències del Cosmos, Universitat de Barcelona (IEEC-UB), Martí i Franquès 1, E-08028 Barcelona, Spain \\ e-mail: nuria.miret-roig@u-bordeaux.fr \\ 2 Laboratoire d'astrophysique de Bordeaux, Univ. Bordeaux, CNRS, B18N, allée Geoffroy Saint-Hilaire, 33615 Pessac, France
}

A\&A, 615, A51 (2018), https://doi.org/10.1051/0004-6361/201731976

Key words. Galaxy: kinematics and dynamics - solar neighborhood - open clusters and associations: general - stars: kinematics and dynamics - stars: formation - errata, addenda

We noticed an error in the original abstract, and provide the corrected version here. The dynamical ages that we obtain are compatible spectroscopic and isochrone fitting ages obtained elsewhere. From the orbital analysis, we suggest a scenario for these YLAs where there were two episodes of star formation: one $\sim 40 \mathrm{Myr}$ ago in the first quadrant that gave birth to Tucana-Horologium, Columba, and
Carina, and another 5-15 Myr ago close to the Sun that formed $\epsilon$ Chamaeleontis, TW Hydrae, and $\beta$ Pictoris. Future Gaia data will provide the necessary accuracy to improve the present results, especially for the controversial age determinations, and additional evidence for the proposed scenario once a complete census of YLAs and better membership can be obtained. 